TPI-MINN-01/38

August 2001

\title{
Phenomemology of a Realistic Accelerating Universe Using Tracker Fields
}

\author{
Vinod B. Johri* \\ Theoretical Physics Institute, School of Physics and Astronomy, \\ University of Minnesota, Minneapolis, MN 55455, USA
}

\begin{abstract}
We present a realistic scenario of tracking of scalar fields with varying equation of state. The astrophysical constraints on the evolution of scalar fields in the physical universe are discussed. The nucleosynthesis and the galaxy formation constraints have been used to put limits on $\Omega_{\phi}$ and estimate $\epsilon$ during cosmic evolution. Interpolation techniques have been applied to estimate $\epsilon \simeq 0.772$ at the present epoch. The epoch of transition from matter to quintessence dominated era and consequent onset of acceleration in cosmic expansion is calculated and taking the lower limit $\Omega_{n}^{0}=0.2$ as estimated from $S N_{e} I_{a}$ data, it is shown that the supernova observations beyond redshift $z=1$ would reveal deceleration in cosmic expansion
\end{abstract}

PACS numbers: 98.80.Cq, 98.80,-k

Emails: vinodjohri@hotmail.com 


\section{Introduction}

The observational view of the universe has drastically changed during the last ten years. Until a decade ago, the universe was supposed to be matter dominated and the cosmic expansion was understood to be slowing down; consequently the Einstein de Sitter model was taken to be the standard model of the observable universe. However, the recent studies undertaken by the Supernova Cosmology Project Team [四] and the High Redshift Search Team [2] reveal that the distant supernovae are fainter and thus more distant than expected for a decelerating universe. It implies that the rate of cosmic expansion is accelerating. This provides empirical evidence of the existence of an exotic matter with repulsive character in the universe which counters gravitational attraction of ordinary matter. If the energy density of such exotic matter is dominant over that of ordinary matter, it may cause acceleration in cosmic expansion. The hypothesis of the existence of exotic matter is in fact supported by the analysis of the luminosity magnitude and redshift measurements of type $I_{a}$ Supernovae that leads to the estimate $\Omega_{n}=0.3 \pm 0.1$ for ordinary matter and $\Omega_{X}=0.7 \mp 0.1$ for exotic matter There are various plausible candidates for the exotic matter, sometimes referred to as dark energy. The Einstein's Cosmological constant $\Lambda$ is a natural choice owing to its repulsive character but it is not acceptable due to its extremely low magnitude compared to particle physics scales. A dynamical cosmological constant with negative pressure looks promising and recently a lot of research has been undertaken to find scalar fields with suitable potentials which roll down slowly to give rise to negative pressure during later stages of evolution and behave like $\Lambda_{\text {effective }}$. Any physical phenomenon, which may cause negative pressure and violate the strong energy condition, would support cosmic acceleration and may be called Quintessence. Various scalar potentials with evolving equation of state have been proposed as probable candidates for quintessence. These include inverse power law, exponential and hyperbolic potential functions [3]-[12] of the scalar field. The growth of scalar fields in the universe is severely restricted by the observational constraints; for instance, the magnitude of $\Omega_{\phi}$ must be low enough so as not to disturb the observed helium abundance at nucleosynthesis $\operatorname{epoch}\left(z=10^{10}\right)$ and not to interfere with the process of formation of galactic structure $(2<z<4)$ but at the same time, the scalar field must be dominant over matter/radiation at the present epoch in order to satisfy the observational estimate of $\rho_{\phi} \sim 2 \rho_{m}$. . This demands extreme fine-tuning of scalar fields relative to matter field. To get rid of fine tuning, the idea of 'tracking ' was put forward [13, 14] and attractor-type potentials were investigated which, irrespective of their divergent initial conditions, evolve along a common track with the background energy density of matter 
and radiation to end up in the domination of $\rho_{\phi}$ over $\rho_{n}$. Whereas, tracking solves the fine tuning and coincidence problems, it does not ensure the compatibility of the scalar field with the observational constraints. Since there is no control over the slow roll down and the rate of growth of the scalar field energy during tracking, the transition to the scalar field dominated phase may take place much later than observed.

The relative growth of the scalar field energy, represented by $\Omega_{\phi}$, may be controlled by monitoring the evolution of the equation of state of the scalar fields through the tracking parameter $\epsilon$ at various observational landmarks in the thermal history of the universe. With this perspective, the concept of 'integrated tracking' was introduced [15] , which links tracking to the estimated value of $\epsilon$ at various epochs during cosmic evolution. The choice of the desired tracker potential is thus narrowed down to those which satisfy the established tracking criterion [15] with $\epsilon$ conforming to the estimated values derived from the observational constraints. This fixes the path of evolution of the tracker field subject to the errors in estimation of $\epsilon$. As shown in this paper $\Omega_{\phi}$ is a function of $\epsilon$ and redshift $z$. Knowing that at the point of transition from matter dominated to the scalar field dominated era $\Omega_{\phi}=0.5, \epsilon=0.666$ and $z=0.414$, we can interpolate the value of $\epsilon$ at other points in cosmic evolution and plot the path of evolution of the tracker field, independent of the choice of tracker potential. In fact, it may not be possible to find a unique tracker potential to cover the entire tracking range but scalar potentials to display tracker behavior under restrictive assumptions may be given.In our previous paper [15], we have traced the paths of tracker potential under the assumption that $\epsilon=$ constant throughout tracking. But it is unrealistic since the value of $\epsilon$ goes on diminishing as you go back in time to the early universe. We present a realistic account of the behavior of tracker potential in this paper by starting with the precisely known values of $\Omega_{\phi}$ and $\epsilon$ at $z=0.414$ and use interpolation methods to estimate $\epsilon=0.772, \Omega_{\phi}=0.66$ at the present epoch $(z=0)$. The astrophysical constraints on $\Omega_{\phi}$ briefly outlined in [15], have been re-examined in this paper and variation

of the tracking parameter, the cosmological density parameter and the equation of state of the scalar field has been plotted in terms of redshift.

\section{Dynamics of Tracking}

Let us first consider CDM cosmology with Quintessence - the rolling scalar fields, with evolving equation of state, which acquire repulsive character (owing to negative pressure) during the late evolution of the universe. The quintessence in the present day observable 
universe, behaves like $\Lambda_{\text {eff }}$ and may turn out to be the most likely form of dark energy which induces acceleration in the cosmic expansion.

Consider the homogeneous scalar field $\phi(t)$ which interacts with matter only through gravity. The energy density $\rho_{\phi}$ and the pressure $p_{\phi}$ of the field are given by

$$
\begin{aligned}
& \rho_{\phi}=\frac{1}{2} \dot{\phi}^{2}+V(\phi) \\
& p_{\phi}=\frac{1}{2} \dot{\phi}^{2}-V(\phi)
\end{aligned}
$$

The equation of motion of the scalar field

$$
\ddot{\phi}+3 H \dot{\phi}+V^{\prime}(\phi)=0, \quad V^{\prime}(\phi) \equiv \frac{d V}{d \phi}
$$

leads to the energy conservation equation

$$
\dot{\rho}_{\phi}+3 H\left(1+w_{\phi}\right) \rho_{\phi}=0
$$

where $w_{\phi} \equiv \frac{p_{\phi}}{\rho_{\phi}}$ and $H \equiv \frac{\dot{a}}{a}$ is the Hubble constant. Accordingly, $\rho_{\phi}$ scales down as

$$
\rho_{\phi} \sim a^{-3\left(1+w_{\phi}\right)}, \quad-1 \leq w_{\phi} \leq 1
$$

Obviously, the scaling of $\rho_{\phi}$ gets slower as the potential energy $V(\phi)$ starts dominating over the kinetic energy $\frac{1}{2} \dot{\phi}^{2}$ of the scalar field and $w_{\phi}$ turns negative.

Since there is minimal interaction of the scalar field with matter and radiation, It follows from Eq.(4) that the energy of matter and radiation is conserved separately as

$$
\dot{\rho}_{n}+3 H\left(1+w_{n}\right) \rho_{n}=0
$$

Accordingly

$$
\rho_{n} \sim a^{-3\left(1+w_{n}\right)}
$$

where $\rho_{n}$ is the energy density of the dominant constituent (matter or radiation) in the universe with the equation of state $p_{n}=w_{n} \rho_{n}$ where $w_{n}=\frac{1}{3}$ for radiation and $w_{n}=0$ for matter.

Although, the scalar field is non-interactive with matter, it affects the dynamics of cosmic expansion through the Einstein field equations. Assuming large scale spatial homogeneity and isotropy of the universe, the field equations for a flat Friedmann model are

$$
H^{2}=\frac{\rho_{n}+\rho_{\phi}}{3 M_{p}^{2}}
$$


and

$$
\frac{2 \ddot{a}}{a}=-\frac{\rho_{n}+\rho_{\phi}+3 p_{n}+3 p_{\phi}}{3 M_{p}^{2}}
$$

where $M_{p}=2.4 \times 10^{18} \mathrm{GeV}$ is the reduced Planck mass.

Denoting the fractional density of the scalar field by $\Omega_{\phi} \equiv \frac{\rho_{\phi}}{\rho_{n}+\rho_{\phi}}$ and that of the matter/radiation field by $\Omega_{n} \equiv \frac{\rho_{n}}{\rho_{n}+\rho_{\phi}}$, equations (8) and (9) may be rewritten as

$$
\Omega_{n}+\Omega_{\phi}=1
$$

and

$$
2 \frac{\ddot{a}}{a}=-\frac{\rho_{n}}{3 M_{p}^{2}}\left[\left(1+3 w_{n}\right)+\left(1+3 w_{\phi}\right) \frac{\Omega_{\phi}}{\Omega_{n}}\right]
$$

The relative growth of $\Omega_{\phi}$ versus $\Omega_{n}$ during the cosmic evolution is given by

$$
\frac{\Omega_{\phi}}{\Omega_{n}}=\frac{\Omega_{\phi}^{0}}{\Omega_{n}^{0}}\left(\frac{a}{a_{0}}\right)^{3 \epsilon}
$$

where the tracking parameter $\epsilon \equiv w_{n}-w_{\phi}$ and $\Omega_{\phi}^{0}, \Omega_{n}^{0}$ denote the values of $\Omega_{\phi}$ and $\Omega_{n}$ at the present epoch $\left(a=a_{0}\right)$. Assuming that that $\Omega_{\phi}^{0} \simeq 2 \Omega_{n}^{0}$, Eq.(12) may be expressed in terms of the red-shift $z$ as below

$$
2\left(\Omega_{\phi}^{-1}-1\right)=(1+z)^{3 \epsilon}
$$

If we insist that the scalar field, regardless of its initial value, should behave like $\Lambda_{\text {eff }}$ today, it must obey tracking conditions [13, 15] which have wide ramifications for quintessence fields already discussed in detail [13, 14, 15]. In nutshell, tracking consists in synchronised scaling of $\rho_{\phi}$ and $\rho_{n}$ along a common evolutionary track so as to ensure the restricted growth of $\Omega_{\phi}$ during the cosmic evolution in accordance with the observational constraints. As discussed in [15], the tracking parameter $\epsilon$ plays a vital role in monitoring the desired growth of $\Omega_{\phi}$. The existence criterion for tracker fields

requires $\epsilon$ to satisfy the condition $\frac{\epsilon \Omega_{n}}{2\left(1+w_{\phi}\right)}<1$ and also to conform to cosmological constraints mentioned therein. Here we investigate the consequent limits on the tracking parameter $\epsilon$ imposed by these constraints to ensure tracking by a quintessence field.

According to the Eq. (13), $\Omega_{\phi}$ depends both upon the redshift $z$ and the tracking parameter $\epsilon$. From a general functional form $\Omega_{\phi}=f(z, \epsilon)$, it is difficult to map out $\Omega_{\phi}-z$ variation through tracking unless we are able to fix the value of $\epsilon$ corresponding to known $\Omega_{\phi}$ at certain points in the phase space of $z-\epsilon$. This is achieved with the help of the 


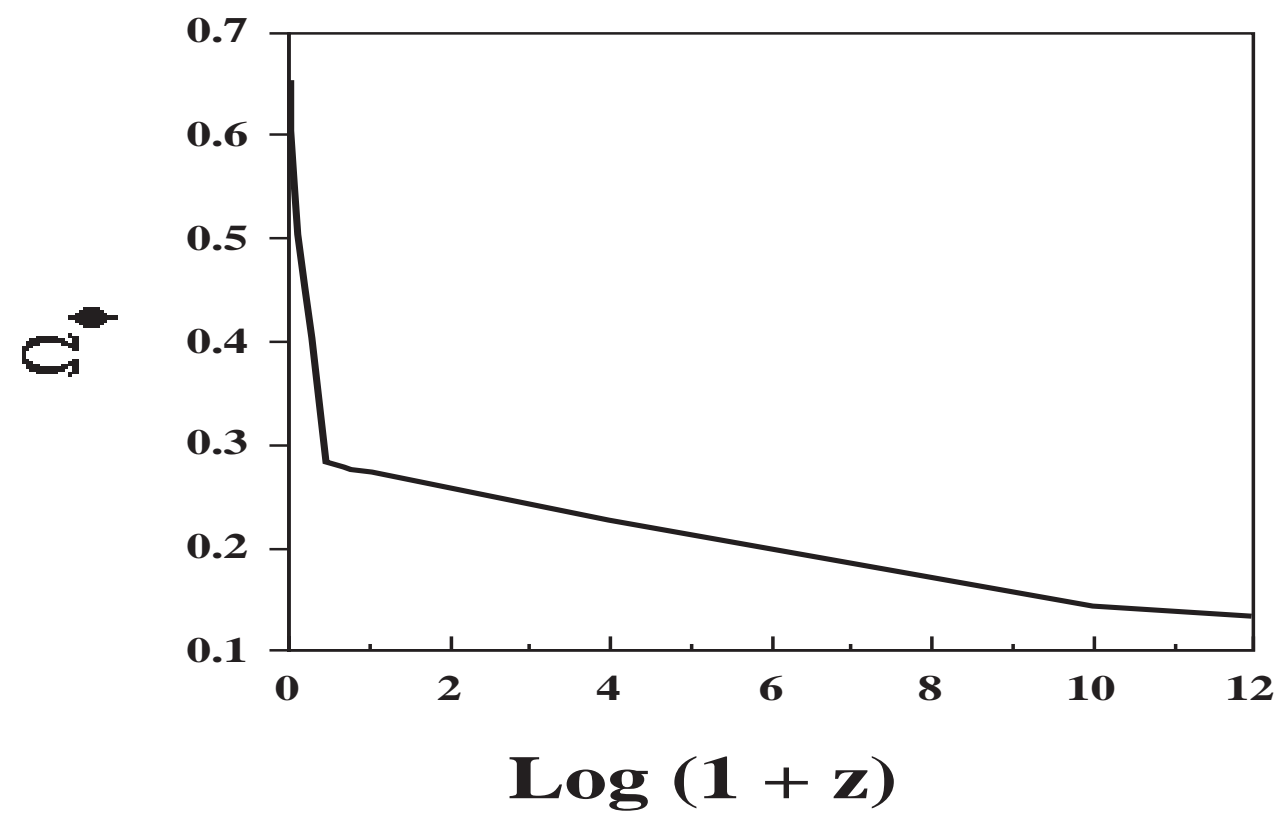

Figure 1: Variation of $\Omega_{\phi}$ versus Redshift $z$ in $Q C D M$ Cosmology assuming $H_{o}=65$ $\mathrm{Km} / \mathrm{Mpc} / \mathrm{s}$.

astrophysical constraints discussed in this paper. Having evaluated $\Omega_{\phi}$ at some typical points $\left(z_{0}, \epsilon_{0}\right)$, we can interpolate $\Delta \Omega_{\phi}$ at neighbouring points $\left(z_{0}+\Delta z_{0}, \epsilon_{0}+\Delta \epsilon_{0}\right)$. Using this technique, realistic tracking diagrams of $\Omega_{\phi}-z$ variation, $w_{\phi}-z$ variation and $\epsilon-z$ variation may be drawn as shown in the figures 1,2 and 3 .

In this connection, the following differential relation, derived from Eq.(13)

$$
-\frac{\Delta \Omega_{\phi}}{3 \epsilon \Omega_{n} \Omega_{\phi}}=\frac{\Delta z}{1+z}+\frac{\Delta \epsilon}{\epsilon} \ln (1+z) .
$$

is found quite useful in interpolating the increment $\Delta \Omega_{\phi}$ in terms of the increments $\Delta z$ and $\Delta \epsilon$. It is noteworthy that the contribution of the term $\frac{\Delta z}{1+z}$ is very small compared to the contribution of $\frac{\Delta \epsilon}{\epsilon}$ at high redshifts.

Let us now reconsider the astrophysical constraints discussed in our previous paper [15], try to refine them and examine their implications for quintessence fields.

1. The Nucleosynthesis Constraint. The first constraint on $\Omega_{\phi}$ during the cosmic evolution comes from the helium abundance at the nucleosynthesis epoch $\left(z \sim 10^{10}\right)$. The presence of an additional component of energy in the form of quintessence field with energy density $\rho_{\phi}$ results in an increase in the value of the Hubble constant $H$ as given by the 


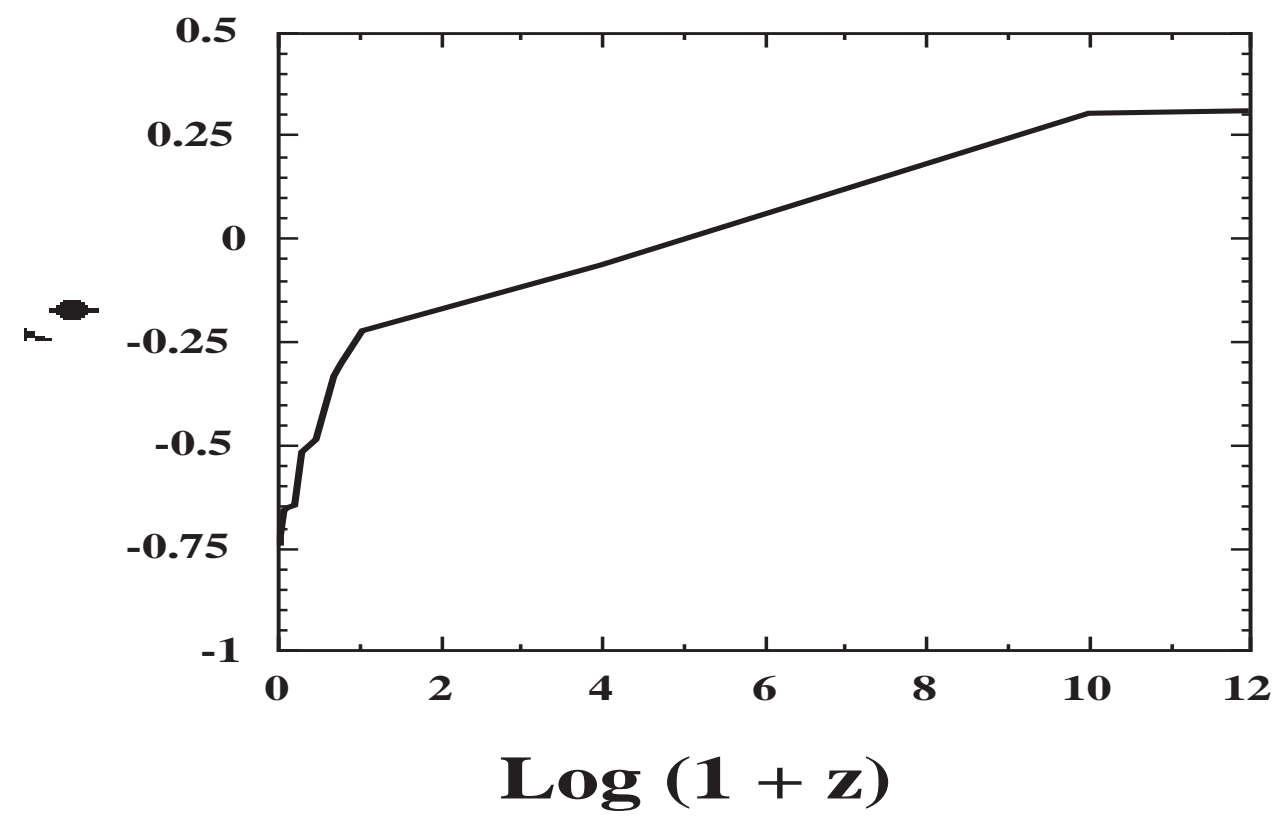

Figure 2: Evolution of the equation of state of the quintessence field in QCDM Cosmology $\left(H_{o}=65 \mathrm{Km} / \mathrm{Mpc} / \mathrm{s}\right)$.

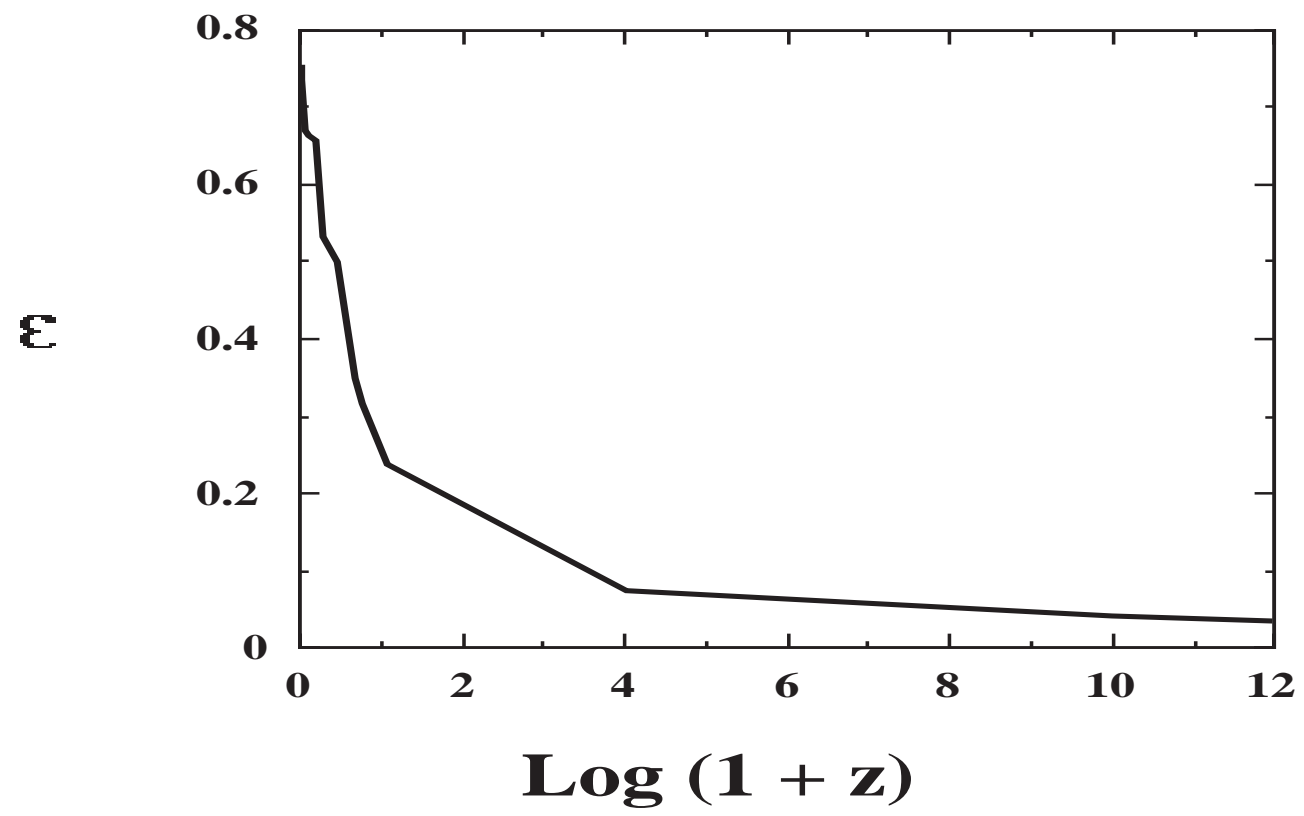

Figure 3: Variation of the tracking parameter $\epsilon$ versus Redshift $z$ in QCDM Cosmology $\left(H_{o}=65 \mathrm{Km} / \mathrm{Mpc} / \mathrm{s}\right)$. 
differential of the Friedmann equation

$$
\frac{2 \delta H}{H}=\frac{\delta \rho}{\rho}=\frac{\rho_{\phi}}{\rho} .
$$

This, in turn, yields a higher ratio of neutrons to protons at the freeze-out temperature $(1 \mathrm{MeV})$ of the weak interactions and a consequent higher percentage of the helium abundance in the universe. Assuming the existence of three known species of neutrinos, the nucleosynthesis calculation [16] yields

$$
\frac{\delta \rho}{\rho}=\frac{7\left(N_{\nu}-3\right)}{43}
$$

Since the number of neutrino species $N_{\nu}<4$, we arrive at the constraint $\frac{\delta \rho}{\rho}<\frac{7}{43}$. If the contribution $\delta \rho$ comes from the quintessence field instead, the above constraint translates into nucleosynthesis constraint on $\Omega_{\phi}$ as follows

$$
\Omega_{\phi}=\frac{\rho_{\phi}}{\rho_{n}+\rho_{\phi}}<\frac{7}{50}=0.14
$$

The corresponding value of the tracking parameter is $\epsilon \leq 0.035$.

2. Galaxy Formation Constraint. According to the current estimates in CDM cosmology, the galactic structure formation takes place between the redshift $z=4$ and $z=$ 2. The clumping of matter into galaxies demands the dominance of gravitational attraction during this period. Therefore, the repulsive force of quintessence must be relatively weak and $\Omega_{\phi}$ must be reasonably less than 0.5 during the galaxy formation era. Interpolation from Eqs. (13) and (14) shows that $0.33 \leq \epsilon<0.5$ during the galaxy formation era.

3. Present Epoch. Two recent surveys [1, 2] based on $S N_{e} I_{a}$ measurements predict accelerating cosmic expansion with $\Omega_{\phi} \simeq 0.7 \pm 0.1$ at the present epoch $(\mathrm{z}=0)$. For the sake of computation, we take the value $\Omega_{\phi}^{0}=0.66$ at $z=0$ and interpolate the values at other redshifts and plot the variation of $\Omega_{\phi}, w_{\phi}$ and $\epsilon$ in terms of $z$ as shown in figures 1,2 and 3 .

The constraint $\ddot{a}>0$ inserted in Eq. (11) leads to $\epsilon>0.5$ at the present epoch. Interpolation with the help of Eq.(14) places $\epsilon$ around 0.77 .

4. Transition to Accelerated Expansion (Quintessence Dominated Era). The onset of acceleration $(\ddot{a} \geq 0)$ in the observable universe takes place around the value of $\Omega_{\phi} \geq 0.5$ which corresponds to $\epsilon \sim 0.666$ from Eq.(11), at a redshift of $z=0.414$ (assuming $\left.\Omega_{n}^{0}=0.33\right)$ from Eq.(13).

In figure 4 , the cosmic events are depicted sequentially by the thick line in the $\Omega_{n}-\Omega_{\phi}$ diagram. The events corresponding to the above constraints are marked by circles. 


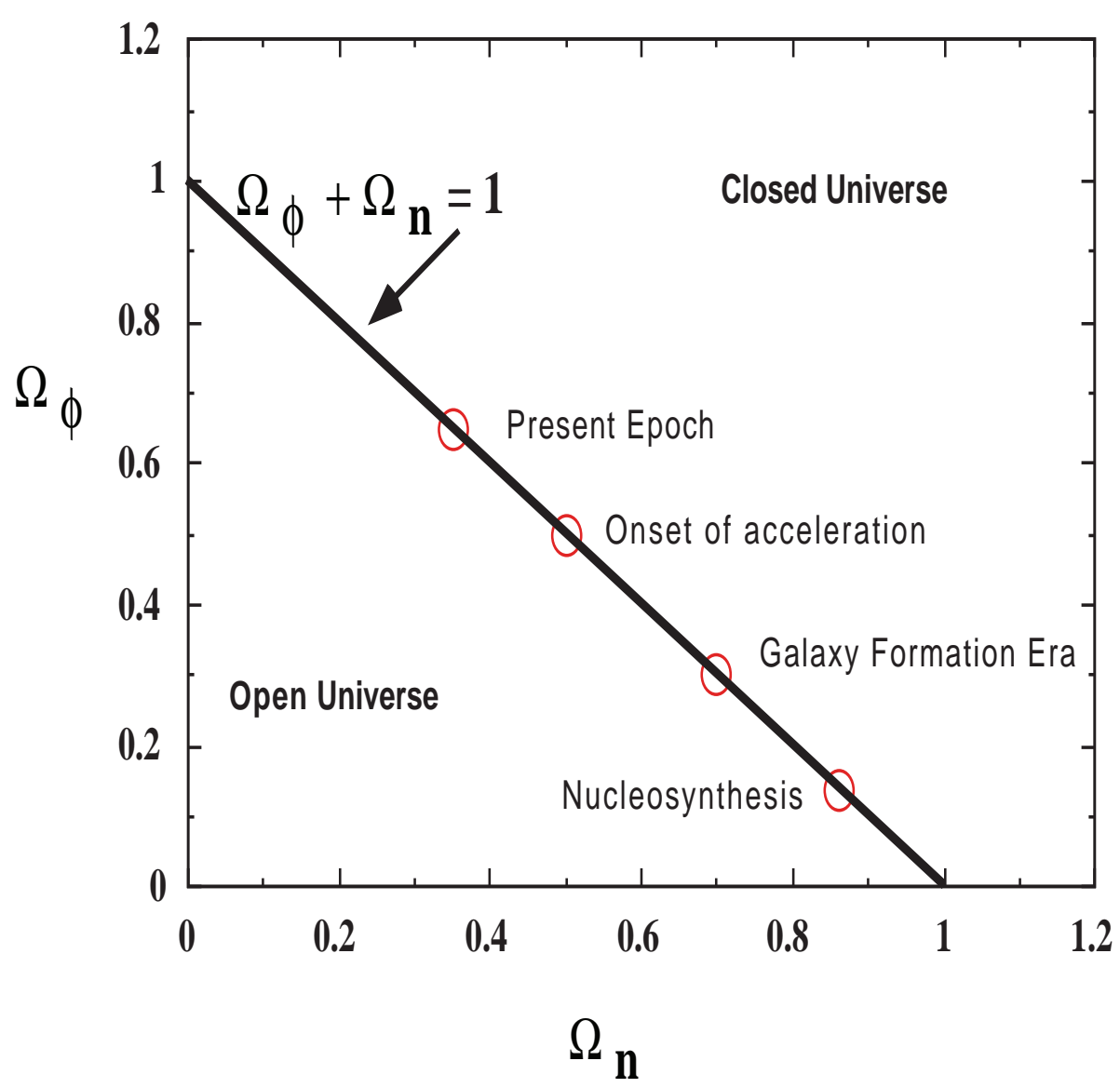

Figure 4: The important cosmic events corresponding to the astrophysical constraints are marked by circles on the thick line (representing spatially flat universe) in QCDM Cosmology. 


\section{Transition to scalar Field Dominated Era}

As revealed by supernova observations, we are living in the quintessence dominated universe today with $\Omega_{\phi} \simeq 2 \Omega_{n}$. When did the transition from the matter to quintessence(scalar field ) dominated era occur? According to tracker field theory, the transition occurs at a stage when $\rho_{\phi} \geq \rho_{n}$ or $\Omega_{\phi} \geq 0.5$ during cosmic evolution. Since $\epsilon=0.666$ at this stage, the transition takes place at a redshift $z^{*}$ as derived from Eq.(12)

$$
z^{*}=\sqrt{\frac{\Omega_{\phi}^{0}}{\Omega_{n}^{0}}}-1
$$

Assuming that the onset of acceleration starts at the transition epoch, the galaxies beyond redshift $z^{*}$ will be found to be decelerating. Taking the lowest value $\Omega_{n}^{0}=0.2$ out of the estimated range from the $S N_{e}$ observations, the largest redshift for transition from Eq. (18) comes out to be $z^{*}=1$. The recent discovery [17] of a type $I_{a}$ supernova at a redshift $z \sim 1.7$ from Hubble Deep Field observations shows the first glimpse of cosmic deceleration It is consistent with the tracker field theory which predicts that the galaxies beyond $z=1$ would have decelerating expansion. This enables us to make another prediction that the future supernovae observations should be able to determine the point of onset of cosmic acceleration and with the knowledge of this redshift, it should be possible to fix the value of $\Omega_{n}^{0}$ and map out the exact course of the future evolution of the universe.

To conclude this paper, we would like to point out that the rolling scalar fields, minimally coupled to matter, seem to be most favourable candidate for the exotic matter (dark energy) in the universe and their behaviour can be completely investigated in the framework of tracker field theory. We have discussed, in this paper, the variation of $\Omega_{\phi}$, the tracking parameter $\epsilon$ and the equation of state parameter $w_{\phi}$ as function of the redshift $z$. Likewise, we can investigate the relative scaling of the energy densities $\rho_{\phi}$ and $\rho_{n}$ as function of $z$ during cosmic evolution, using Eqs. (7) and (13).

Acknowledgments This work was supported by UGC grant from India. The author acknowledges useful discussions and valuable help of Keith Olive and Panagiota Kanti and hospitality of Theoretical Physics Institute, University of Minnesota.

* Permanent Address: Department of Mathematics and Astronomy, Lucknow University, Lucknow 226007. India. 


\section{References}

[1] S.Perlmutter et al, bull.Am.Astron Soc. 29,1351 (1997), Astrophys. J. 517, 565(1999) (1998).

[2] A.G.Riess et al, Astron.J. 116, 1009 (1998). P.Garnavich et al, Astrophysical J. 493, L53 (1998) Astrpphysical J.509,74(1998) B.P.Schmidt et al, ibid,507,46(1998)

[3] P.J.E.Peebles and B.Ratra, Astrophysical. J.Lett.325,L17 (1998). B.Ratra abd P.J.E.Peebles,Phys.Rev.D 37,3406(1998)

[4] C.Wetterich,Nucl. Phys. B 302, 668 (1988).

[5] L.P.Chimento and A.S.Jakubi,Int J.Mod.Phys.5, 71(1996).

[6] P.G.Ferreira and M.Joyce,Phys.Rev D,58, 023503(1998).

[7] T,Matos, F.S. Guzman and L.A.Weena-Lopez,Class, Quantum Grav. 17, 1707 (2000).

[8] T.Barrieiro, E.J.Copeland and N.J.Nunes, Phys.Rev.D. 61 127301(2000)

[9] A.de la Macorra and G.Piccilnelli,Phys.Rev.D 61, 123503(2000)

[10] R.R. Coldwell,R.Dave, and P.J.Steinhardt,phys.Rev.Lett 80, 1582 (1998)

[11] A.R.Liddl and R.J.Scherrer, Phys. Rev.D 59, 023509 (1999)

[12] D. Wands,E.J.Copeland and A.R.Liddle,Phys, Rev. D57,4686 (1998)

[13] I. Zlatev, L. Wang and P.J. Steinhardt, Phys. Rev. Lett. 82, 896 (1999).

[14] P.J. Steinhardt et al, Phys. Rev. D 59, 123504 (1999).

[15] V.B. Johri, Phys. Rev.D 63,103504 (2001)

[16] K.A. Olive and D. Thomas, Astroparticle Physics 11, 403(1999).

[17] A.G.Riess et al, astro-ph/0104455 v1 (27 April,2001) 